${ }^{1}$ Hospital San José. Fundación

Universitaria de Ciencias de la

Salud. Bogotá, Colombia.

${ }^{2}$ Organización Sanitas (Keralty)/

Clínica Colsanitas. Bogotá, Colombia.

${ }^{3}$ Hospital Pablo Tobón Uribe.

Medellín, Colombia.

${ }^{4}$ Hospital La Misericordia. Bogotá, Colombia.

${ }^{5}$ NeuroEconomix. Bogotá, Colombia.

Los autores declaran no tener conflictos de interés.

Trabajo recibió financiamiento de

Novo Nordisk Colombia.

Recibido el 2 de junio de 2018, aceptado el 27 de diciembre de

2018.

Correspondencia a:

Linda Ibatá Bernal

lindaibata@gmail.com

\section{Importancia de la orientación diagnóstica en hemofilia $A$ adquirida}

\author{
CLAUDIA PATRICIA CASAS PATARROYO ${ }^{1}$, CLAUDIA DEL PILAR \\ AGUDELO LÓPEZ ${ }^{2}$, KENNY GALVEZ ${ }^{3}$, JIMMY LAGOS IBARRA ${ }^{4}$, \\ SUSAN MARTÍNEZ ROJAS ${ }^{5}$, LINDA IBATÁ BERNAL ${ }^{5}$
}

\section{Adequate diagnosis of acquired hemophilia A}

Acquired hemophilia A (AHA) is a rare and life-threatening autoimmune hemorrhagic disorder where autoantibodies are developed against factor VIII. An early diagnosis is challenging and mandatory: an immediate hemostatic control is required to reduce morbidity and mortality. Laboratory features of AHA are: presence of autoantibodies against factor VIII, prolonged activated partial thromboplastin time (with normal prothrombin time and thrombin time) and decreased factor VIII levels. In some cases, the results of laboratory tests may be incorrect due to errors in analysis, blood extraction or manipulation of samples; also worth of consideration are limitations in the measurement range and low sensitivity of the tests. This review highlights the importance of adequate screening in patients with suspected AHA to make an adequate diagnosis and reduce overall fatal outcomes.

(Rev Med Chile 2019; 147: 334-341)

Key words: Diagnosis; Factor VIII; Hemophilic A; Hemorrhagic Disorders.
L a hemofilia A adquirida (HAA) es un trastorno hemorrágico mediado por un proceso autoinmune, en el que se desarrollan anticuerpos contra el factor VIII de la coagulación. Esto altera la función hemostática y se presenta con manifestaciones hemorrágicas, las cuales potencialmente pueden amenazar la $v \operatorname{via}^{1}$. Puede haber aparición súbita de sangrados en piel y sangrados subcutáneos diseminados (en el $80 \%$ de los casos), en ausencia de antecedentes personales y familiares de alteraciones en la coagulación ${ }^{1}$.

La HAA es poco frecuente, con una incidencia de 1-1,5 casos por un millón de personas por año $0^{2-5}$. Su incidencia es similar en diferentes grupos étnicos ${ }^{6}$. La incidencia de AHA aumenta con la edad, con una incidencia anual estimada en 0,045 casos por millón en menores de 16 años ${ }^{7}$. La edad de aparición más frecuente se describe en adultos jóvenes (20 a 40 años), especialmente mujeres en período postparto (incidencia $1 / 350.000$ partos $)^{8}$ con enfermedades autoinmu- nes $^{9} \mathrm{o}$ en personas de ambos sexos en promedio a los 68-70 años ${ }^{10,11}$.

Hay diferentes retos en el diagnóstico de esta patología. Dada su baja incidencia, la sospecha clínica y el diagnóstico pueden ser difícil de sospechar. A parte de esto la selección de pruebas diagnósticas adecuadas y la técnica de obtención y procesamiento de las muestras sanguíneas pueden producir errores en la interpretación de los mismos $^{12}$. Todos estos errores corresponden a errores de tipo pre y analíticos. Estos pueden producir los retrasos en el diagnóstico, contribuyen a la tasa de mortalidad de estos pacientes ${ }^{2}$.

Esta revisión presenta un resumen sobre la importancia del diagnóstico en la HAA y las posibles dificultades que se presentan con respecto a las pruebas diagnósticas, principalmente, el proceso previo a la aplicación y análisis de las pruebas (pre-analítica). De esta manera, se espera que médicos y laboratorios de procesamiento de muestras puedan tener estas consideraciones en cuenta con el fin de reducir errores diagnósticos ${ }^{12}$. 


\section{Metodología}

Se realizó una revisión panorámica de la literatura acerca de hemofilia A adquirida, priorizando estudios relacionados con epidemiología, fisiopatología, diagnóstico, limitaciones dificultades en el diagnóstico. Se llevó a cabo una búsqueda en las bases de datos Medline, Embase, Lilacs y Cochrane Database of Systematic Reviews y una búsqueda en bola de nieve a partir de los estudios seleccionados. Se incluyó información de 32 referencias, teniendo en cuenta guías de práctica clínica y revisiones de tema, publicadas en inglés o español, entre 2012 y 2017.

\section{Fisiopatología de la hemofilia A adquirida}

En la hemostasia normal, los factores VIIIa y IXa forman el complejo tenasa sobre la superficie plaquetaria. Este activa el factor $\mathrm{X}$, que se une al factor $\mathrm{Va}$ (complejo de protrombinasa) para transformar protrombina (factor II) en trombina (factor IIa) (Figura 1 izquierda) ${ }^{13}$. En la HAA (Figura 1 derecha), la acción inhibitoria contra el factor VIII (auto anticuerpos tipo inmunoglobulinas $\mathrm{G}$ inhibitorias, generalmente de los subtipos IgG1 e IgG4) impide la formación del complejo tenasa activado en la superficie plaquetaria, lo que conduce a un fallo en la generación de trombina y, consecuentemente, a la formación de coágulos de fibrina inestables ${ }^{1,11,13-15}$.

Como parte de la cinética de los auto anticuerpos contra el factor VIII en HAA, se observa una reacción inicial en la que no neutralizan completamente al factor VIII, por lo que es po- sible identificar actividad residual de este factor VIII en presencia del inhibidor. Por lo anterior, la actividad residual de factor VIII no se correlaciona con los títulos de inhibidores ni con la tendencia al sangrado ${ }^{1,16,17}$. La pérdida de auto tolerancia al factor VIII subyace en el desarrollo de los auto-anticuerpos. Se ha sugerido un papel de factores genéticos (genes de respuesta inmune y genotipo HLA) ya que se ha observado una asociación entre la incidencia de HAA y la presencia de un polimorfismo del antígeno 4 del linfocito $\mathrm{T}$ citotóxico o expresión de los alelos HLA DRB1 ${ }^{\star} 16$ y DQB $1^{\star} 0502^{18}$. Por lo anterior, se considera que existe una predisposición genética como factor asociado a la patogénesis de esta condición, sin descartar factores ambientales.

\section{Diagnóstico}

La HAA debe sospecharse en pacientes con:

- Sangrado anormal de inicio reciente, con prolongación del tiempo de tromboplastina parcial activada (TTPa) y con tiempo de protrombina normal ${ }^{19}$.

- Pacientes que no presentan manifestaciones de sangrado con prolongación aislada del tiempo parcial de tromboplastina activada ${ }^{19}$.

- Pacientes con una prueba de mezclas consistente con presencia de un inhibidor específico, y con anticoagulante lúpico negativo, sin evidencia de uso de anticoagulantes y otros factores que alteren las pruebas de coagulación ${ }^{19}$.

- Se debe considerar especialmente la sospecha en pacientes ancianos y en mujeres periparto y posparto ${ }^{19}$.

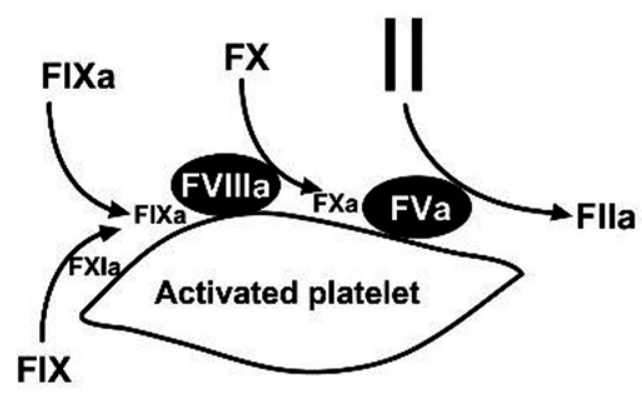

$1 B$

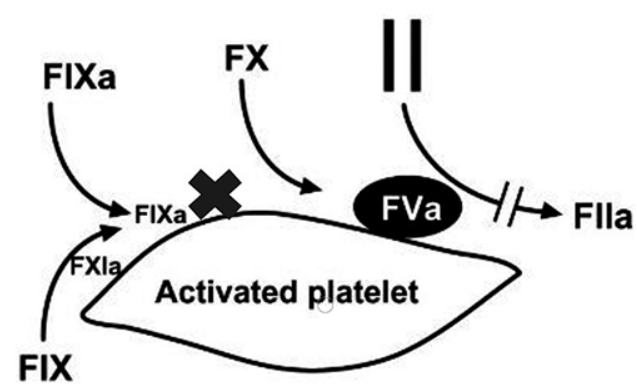

Figura 1. Fisiología de la coagulación y fisiopatología de la hemofilia A adquirida. Tomado de Hoffman et al'13. 
La HAA tiene un perfil de laboratorio característico:

- presencia de auto anticuerpos contra el factor VIII;

- tiempo parcial de tromboplastina activada prolongado (con tiempo de protrombina y tiempo de trombina normales);

- niveles de factor VIII disminuidos ${ }^{9,19}$.

Un algoritmo de orientación diagnóstica se describe en la Figura 2. El estudio de TTPa prolongado inicia con la confirmación de dicho resultado mediante repetición del TTPa, y la exclusión de tratamiento anticoagulante o administración de otros medicamentos o productos que pueden interferir en la coagulación.

La prueba de mezclas es el método de laboratorio de primera línea para investigar la causa de una prolongación del TTPa. Esta prueba consiste en combinar el plasma del paciente con plasma normal, para luego evaluar si el tiempo de coagulación se normaliza o permanece prolongado. La prueba de mezclas tiene dos momentos: la inmediata y la incubada. En la prueba de mezclas inmediata el tiempo parcial de tromboplastina activada se realiza inmediatamente después de mezclar el plasma del paciente y el plasma normal. En la prueba de mezclas incubada, el tiempo parcial de tromboplastina activada se realiza después de incubar la mezcla de plasma durante una a dos horas a $37^{\circ} \mathrm{C}^{20}$.

La interpretación de la prueba de mezclas se puede dar en los siguientes escenarios:

- En una muestra con déficit de factores de coagulación, las mediciones inmediata e incubada darán un TTPa corregido. Por lo que se procede a cuantificar factores específicos para identificar el déficit.

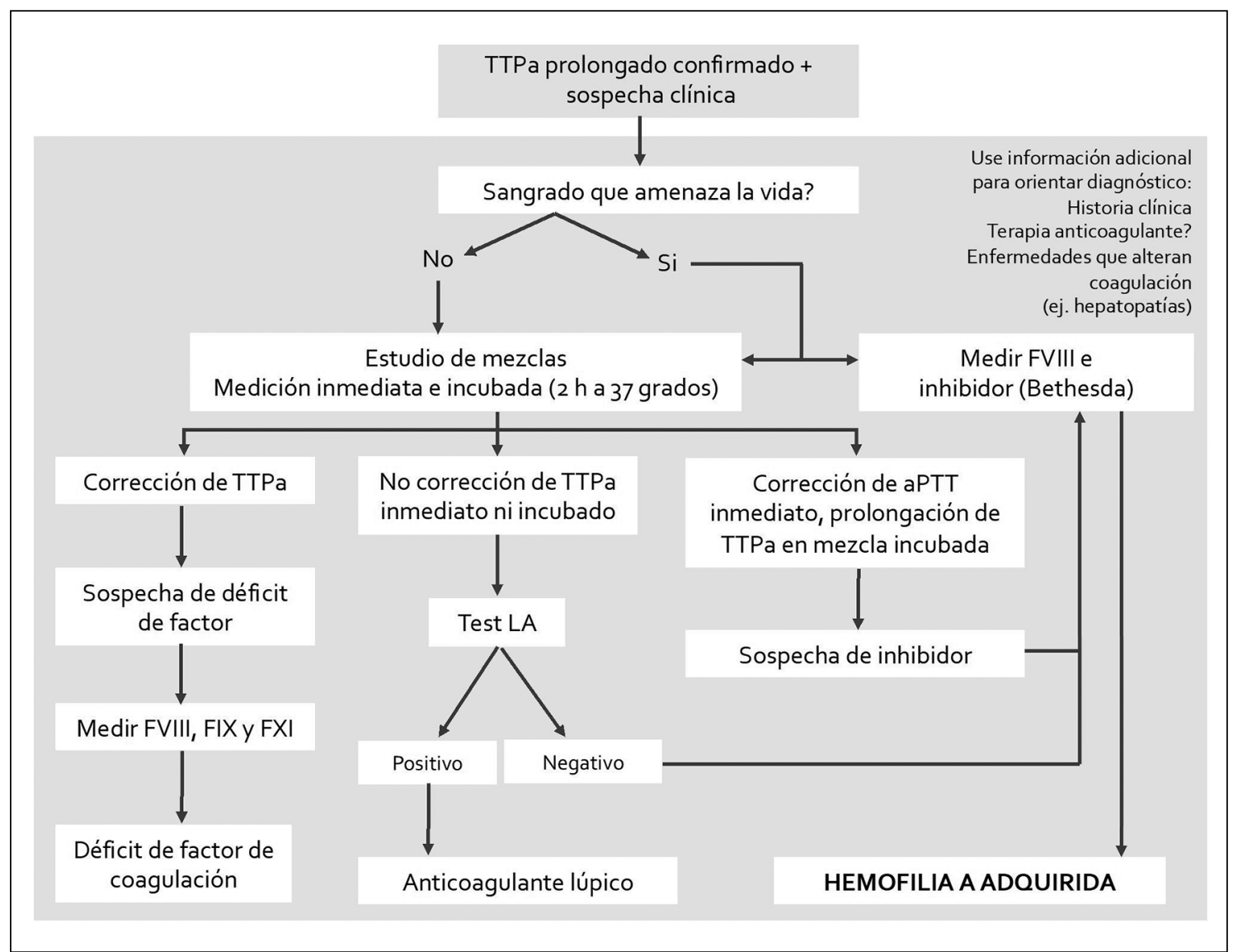

Figura 2. Algoritmo diagnóstico de laboratorio de hemofilia A adquirida. 
- Las muestras con inhibidores de factor de reacción rápida no corregirán la prueba de mezclas inmediata, ni en la fase incubada. Se ampliará el estudio con pruebas de anticoagulante lúpi$\mathrm{CO}^{21,22}$.

- Las muestras con inhibidores de reacción lenta o dependientes del tiempo (la mayoría de los inhibidores del factor VIII y $8 \%$ de los anticoagulantes lúpicos) no corregirán el TTPa en la prueba incubada, o pueden corregir la mezcla inmediata, pero en una medición posterior evidenciarán la presencia del inhibidor ${ }^{23-25}$. Se procederá a medición de título de inhibidores.

- El inhibidor es cuantificado en Unidades Bethesda, por el método Bethesda clásico o por método Nijmegen Bethesda (recomendada por la ISTH $)^{15}$, definiéndose 1 unidad Bethesda por $\mathrm{mL}(\mathrm{UB} / \mathrm{mL})$ la que corresponde a la cantidad de inhibidor capaz de bloquear $50 \%$ del factor VIII circulante ${ }^{11}$. Las $\mathrm{UB} / \mathrm{mL}$ son la convención internacional establecida para definir la actividad de un inhibidor y se considera que tendrá relevancia clínica cuando su título sea $\geq 0,6 \mathrm{UB} / \mathrm{mL}^{22}$.

Las dificultades en el correcto diagnóstico o en la adecuada exclusión de HAA pueden deberse a aspectos analíticos. Sin embargo, los problemas pre-analíticos actúan como un factor determinante en la precisión y calidad de los resultados, aunque generalmente son poco reconocidos, dado que involucran pasos previos a la participación del laboratorio que realiza las pruebas ${ }^{12}$. Desconociendo estas potenciales limitantes, tanto el laboratorio que entrega el resultado, como el clínico, podrán incurrir en conductas basadas en valores imprecisos.

\section{Aspectos pre-analíticos de importancia}

En las pruebas de mezclas, así como en las de coagulación se deben evitar errores de la fase pre analítica (toma y manejo de muestras) ${ }^{21}$, que pueden presentarse y conducir a resultados inexactos y errores diagnósticos. La realización de pruebas de coagulación requiere conocimiento y experiencia, con las consideraciones siguientes:

- La toma de la muestra sanguínea debe realizarse cerca del laboratorio para asegurar su rápido traslado. Es importante resaltar que lo más importante es la adecuada manipulación y traslado en condiciones que aseguren la preservación de la muestra.

- Realizar la venopunción con la técnica recomendada y recolectar la muestra en tubos citratados.

- Las muestras deben conservarse a una temperatura entre $20^{\circ} \mathrm{C}$ y $25^{\circ} \mathrm{C}$ (temperaturas diferentes afectan la actividad del factor VIII) y ser analizadas dentro de las cuatro horas de realizada la extracción ${ }^{21}$.

- Seguir siempre los lineamientos de alta calidad de procesamiento de muestras de coagulación.

La extracción de sangre es un problema pre-analítico que puede conducir a errores en diagnóstico de HAA. Otros elementos a considerar son las características de paciente el procesamiento de la muestra extraída y los instrumentos de fabricantes con los cuales se evaluarán las muestras.

Muchos errores pre-analíticos se basan en la extracción de la muestra de sangre. Desafortunadamente, no siempre hay suficiente claridad de cuando una muestra es pobre o inadecuada para el análisis ${ }^{26}$. Aspectos tan sencillos como la identificación errónea del paciente y error en el etiquetado de los tubos, resultará en interpretaciones inadecuadas de los resultados ${ }^{12}$. Para poder identificar con mayor facilidad las principales situaciones problemáticas pre-analíticas relacionadas con la extracción de la muestra de sangre, éstas se señalan en la Tabla 1.

Un aspecto pre-analítico que puede afectar el resultado de las pruebas diagnósticas se configura en los pacientes bajo terapia de anticoagulación con trastornos hemorrágicos (Tabla 2) ${ }^{12}$. La importancia radica en que, una vez iniciado el tratamiento, probablemente se tendrán bajos niveles de factores VIII y IX ${ }^{27,28}$.

Por último, hay 3 condiciones estándar que representan los aspectos pre-analíticos más comunes, los cuales se centran en los instrumentos desarrollados por fabricantes para evaluar las pruebas. Estos tres eventos (hemólisis/ictericia/lipemia) generan cambios en la transmisión y absorción de la luz y, por lo tanto, afectan cualquier prueba que dependa de la densidad óptica o lecturas de turbidez (especialmente relevante para los ensayos de coágulos ejecutados en analizadores ópticos). Esto puede conllevar a un diagnóstico errado de HAA (Tabla 3). 


\section{Tabla 1. Resumen de los principales errores en el momento de la toma de muestra que dificultan la correcta identificación de Hemofilia A adquirida}

\begin{tabular}{|c|c|c|c|}
\hline Evento relacionado & Efecto en la muestra & Efecto en la prueba & Solución \\
\hline $\begin{array}{l}\text { 1. Uso excesivo del } \\
\text { torniquete o estasis } \\
\text { excesiva }\end{array}$ & - Hemoconcentración & $\begin{array}{l}\text { - Alteración en las pruebas } \\
\text { de TTPa }\end{array}$ & $\begin{array}{l}\text { - Toma de muestras de } \\
\text { sangre según guías de } \\
\text { práctica }{ }^{28}\end{array}$ \\
\hline $\begin{array}{l}\text { 2. Aguja demasiado } \\
\text { pequeña }\end{array}$ & - Hemólisis & & $\begin{array}{l}\text { - Educación de personal } \\
\text { encargado de toma de } \\
\text { muestra } 29,30\end{array}$ \\
\hline $\begin{array}{l}\text { 3. Tubos para pruebas } \\
\text { de coagulación bajo la } \\
\text { capacidad indicada }\end{array}$ & $\begin{array}{l}\text { - Dilución y sobrecitración: } \\
\text { exceso de anticoagulación } \\
\text { del plasma }\end{array}$ & $\begin{array}{l}\text { - Falsa disminución de } \\
\text { factores medibles (VIII, } \\
\text { IX), por lo tanto, falso } \\
\text { diagnóstico de AHA }\end{array}$ & $\begin{array}{l}\text { - Entrenar al personal } \\
\text { encargado de toma de }\end{array}$ \\
\hline $\begin{array}{l}\text { 4. Tubos para pruebas de } \\
\text { coagulación sobre la } \\
\text { capacidad indicada }\end{array}$ & $\begin{array}{l}\text { - Dificultad en la mezcla } \\
\text { - Coagulación parcial o } \\
\text { completa de la muestra }\end{array}$ & $\begin{array}{l}\text { - Falsa disminución de fac- } \\
\text { tores medibles (VIII, IX), } \\
\text { por lo tanto, se considera } \\
\text { un diagnóstico falso de } \\
\text { AHA si la muestra se coa- } \\
\text { gula completamente } \\
\text { - Falsa elevación de factor } \\
\text { VIII, si la coagulación par- } \\
\text { cial conduce a activación } \\
\text { de muestra }\end{array}$ & $\begin{array}{l}\text { muestra de la importancia } \\
\text { de que los tubos de } \\
\text { sangre citratados no } \\
\text { sobrepasen la marca }{ }^{27} \\
\text { - Educación de personal } \\
\text { encargado de toma de } \\
\text { muestra } 29,30\end{array}$ \\
\hline $\begin{array}{l}\text { 5. Plasma con EDTA o } \\
\text { heparinizado }\end{array}$ & $\begin{array}{l}\text { - EDTA y heparina actúan } \\
\text { como inhibidor de factores } \\
\text { medibles } \\
\text { - Elevación de TTPa } \\
\text { - Prueba de mezclas inade- } \\
\text { cuada } \\
\text { - Falsa disminución y falsos } \\
\text { inhibidores de factores } \vee \text { y VIII }\end{array}$ & - Falso diagnóstico de AHA & $\begin{array}{l}\text { - No use plasma con EDTA } \\
\text { o heparina para investigar } \\
\text { hemofilia } \\
\text { - Se puede identificar } \\
\text { presencia de EDTA por } \\
\text { pruebas bioquímicas } \\
\text { - Se puede identificar } \\
\text { heparina por pruebas } \\
\text { anti-Xa }\end{array}$ \\
\hline 6. Suero & $\begin{array}{l}\text { - TTPa descartable } \\
\text { - Falsa disminución de } \\
\text { factores II, V, VIII, IX, X }\end{array}$ & - Falso diagnóstico de AHA & $\begin{array}{l}\text { - No use suero para } \\
\text { investigar hemofilia } \\
\text { - Se puede identificar } \\
\text { suero con pruebas de } \\
\text { fibrinógeno }\end{array}$ \\
\hline $\begin{array}{l}\text { 7. Alto hematocrito en } \\
\text { muestras }\end{array}$ & $\begin{array}{l}\text { - Prolongación de TP y TTP } \\
\text { - Reducción de todos los } \\
\text { factores, incluyendo VIII, IX }\end{array}$ & - Falso diagnóstico de AHA & $\begin{array}{l}\text { - Realizar ajustes de citrato } \\
\text { si es necesario }{ }^{27}\end{array}$ \\
\hline
\end{tabular}

TTPa: Tiempo de tromboplastina parcial activado. EDTA: Ácido etilendiaminotetra-Acético. Elaboración propia en base a referencias: Favaloro EJ, Lippi G. Preanalytical issues that may cause misdiagnosis in haemophilia and von Willebrand disease. Haemophilia 2017; 1-13. Favaloro EJ, Lippi G, Adcock DM. Preanalytical and postanalytical variables: the leading causes of diagnostic error in hemostasis? Semin Thromb Hemost. 2008; 34 (7): 612-34. Lippi G, Favaloro EJ. Preanalytical issues in hemostasis and thrombosis testing. Methods Mol Biol 2017; 1646: 29-42. Lippi G, Salvagno GL, Montagnana M, Lima-Oliveira G, Guidi GC, Favaloro EJ. Quality standards for sample collection in coagulation testing. Semin Thromb Hemost 2012; 38: 565-75. 
Tabla 2. Resumen de los principales errores que dificultan la correcta identificación de Hemofilia A adquirida, relacionados con el tratamiento anticoagulante como aspecto pre-analítico

\begin{tabular}{|c|c|c|}
\hline Evento relacionado & Consecuencia & Solución \\
\hline $\begin{array}{l}\text { 1. Paciente heparinizado } \\
\text { investigado por evento } \\
\text { de sangrado }\end{array}$ & $\begin{array}{l}\text { - La heparina actúa como un inhibidor de } \\
\text { factores } \\
\text { - TTPa elevado } \\
\text { - Prueba de mezclas inadecuada } \\
\text { - Falsa disminución y falsos inhibidores de } \\
\text { factores VIII, IX, XI, XII }\end{array}$ & $\begin{array}{l}\text { - Como médico tratante, ser cuidadoso al } \\
\text { investigar pacientes heparinizados para } \\
\text { eventos hemorrágicos } \\
\text { - La heparina se puede identificar por } \\
\text { pruebas anti-Xa }\end{array}$ \\
\hline $\begin{array}{l}\text { 2. Paciente con anticoagulantes } \\
\text { orales investigado por evento } \\
\text { de sangrado }\end{array}$ & $\begin{array}{l}\text { - Niveles reducidos de la mayoría de los } \\
\text { factores de coagulación } \\
\text { - Patrón de prueba de tipo inhibitorio }\end{array}$ & $\begin{array}{l}\text { - Medición en sangre de anticoagulantes } \\
\text { orales, para evaluar niveles sanguíneos } \\
\text { del fármaco }\end{array}$ \\
\hline
\end{tabular}

TTPa: Tiempo de tromboplastina parcial activado. Elaboración propia en base a referencias: Favaloro EJ, Lippi G. Preanalytical issues that may cause misdiagnosis in haemophilia and von Willebrand disease. Haemophilia 2017; 1-13. Favaloro EJ, Lippi G, Adcock DM. Preanalytical and postanalytical variables: the leading causes of diagnostic error in hemostasis? Semin Thromb Hemost 2008; 34 (7): 612-34. Lippi G, Favaloro EJ. Preanalytical issues in hemostasis and thrombosis testing. Methods Mol Biol 2017; 1646: 29-42. Lippi G, Salvagno GL, Montagnana M, Lima-Oliveira G, Guidi GC, Favaloro EJ. Quality standards for sample collection in coagulation testing. Semin Thromb Hemost 2012; 38: 565-75.

Tabla 3. Resumen de instrumentos de fabricantes, relacionados con aspectos pre-analíticos que dificultan el diagnóstico de AHA. Efecto de características de las muestras sobre las pruebas de coagulación

\begin{tabular}{|c|c|c|c|c|}
\hline \multicolumn{5}{|c|}{ Instrumentos de fabricantes } \\
\hline $\begin{array}{l}\text { Evento } \\
\text { relacionado }\end{array}$ & Consecuencia & Significado & $\begin{array}{l}\text { Frecuencia } \\
\text { del evento }\end{array}$ & Solución \\
\hline 1. Hemólisis & $\begin{array}{l}\text { - Efectos espectrales } \\
\text { en los ensayos sobre } \\
\text { coágulos (se afectará } \\
\text { la lectura de densidad } \\
\text { óptica) } \\
\text { - Efectos biológicos de } \\
\text { relastasas de células; } \\
\text { efectos de posible } \\
\text { activación }\end{array}$ & $\begin{array}{l}\text { - Los resultados de } \\
\text { las pruebas estarán } \\
\text { sesgados, de tipo } \\
\text { ensayo- específico }\end{array}$ & Frecuente & $\begin{array}{l}\text { - Revisar sistemáticamente } \\
\text { la calidad de la muestra } \\
\text { - No realizar análisis } \\
\text { cuando la interferencia es } \\
\text { clínicamente significativa } \\
\text { - Tomar otra muestra }\end{array}$ \\
\hline 2. Ictericia & $\begin{array}{l}\text { - Efectos espectrales } \\
\text { en los ensayos sobre } \\
\text { coágulos (se afectará } \\
\text { la lectura de densidad } \\
\text { óptica) }\end{array}$ & $\begin{array}{l}\text { - Los resultados de } \\
\text { las pruebas estarán } \\
\text { sesgados, de tipo } \\
\text { ensayo- específico }\end{array}$ & No común & $\begin{array}{l}\text { - Revise sistemáticamente } \\
\text { la calidad de muestra } \\
\text { - No realizar análisis } \\
\text { cuando la interferencia es } \\
\text { clínicamente significativa }\end{array}$ \\
\hline 3. Lipemia & $\begin{array}{l}\text { - Efectos espectrales } \\
\text { en los ensayos sobre } \\
\text { coágulos (afectarán las } \\
\text { lecturas de densidad } \\
\text { óptica). Desplazamiento } \\
\text { de elementos del plasma }\end{array}$ & $\begin{array}{l}\text { - Los resultados de } \\
\text { las pruebas estarán } \\
\text { sesgados, de tipo } \\
\text { ensayo- específico }\end{array}$ & No común & $\begin{array}{l}\text { - Revise sistemáticamente } \\
\text { la calidad de muestra } \\
\text { - No realizar análisis } \\
\text { cuando la interferencia es } \\
\text { clínicamente significativa }\end{array}$ \\
\hline
\end{tabular}

Elaboración propia en base a referencias: Favaloro EJ, Lippi G. Preanalytical issues that may cause misdiagnosis in haemophilia and von Willebrand disease. Haemophilia 2017; 1-13. Favaloro EJ, Lippi G, Adcock DM. Preanalytical and postanalytical variables: the leading causes of diagnostic error in hemostasis? Semin Thromb Hemost. 2008; 34 (7): 612-34. Lippi G, Favaloro EJ. Preanalytical issues in hemostasis and thrombosis testing. Methods Mol Biol 2017; 1646: 29-42. Lippi G, Salvagno GL, Montagnana M, Lima-Oliveira G, Guidi GC, Favaloro EJ. Quality standards for sample collection in coagulation testing. Semin Thromb Hemost 2012; 38: 565-75. 


\section{Discusión}

La HAA es una patología infrecuente, con un cuadro clínico configurado por equimosis, hematomas extensos, anemia y sangrados graves pueden configurar un espectro de gravedad de presentación clínica, que si no se controla puede tener desenlaces fatales.

Su diagnóstico puede tener dificultades, aunque puede ser facilitado al tener en cuenta los grupos etáreos de mayor incidencia, la disponibilidad de las pruebas para realizar un diagnóstico acertado, el adecuado manejo de muestras de sangre y el análisis e interpretación adecuados de las pruebas de laboratorio.

Los diferentes trastornos de la coagulación pueden presentar síntomas similares y, por esta razón, deberán evaluarse de manera integral los posibles diagnósticos diferenciales. Los pacientes con síntomas de sangrado reciente deben ser evaluados considerando la posibilidad de autoinmunidad de otro tipo, trastornos de sangrado congénito, trastornos de sangrado adquirido y efectos de medicamentos como heparinas, heparinoides y anticoagulantes directos. Las pruebas de laboratorio deben realizarse en medio de las condiciones técnicas de mayor calidad que aseguren un diagnóstico preciso ${ }^{26}$.

Los problemas preanalíticos y analíticos en las pruebas de hemostasia son una causa importante de error de diagnóstico y pueden conducir a eventos clínicos adversos significativos ${ }^{26}$. La robustez de los instrumentos modernos ha disminuido sustancialmente el riesgo de errores analíticos y, por lo tanto, los resultados de las pruebas se han hecho más precisos. Sin embargo, la fase preanalítica puede conllevar a situaciones donde las pruebas pierden validez y eso puede tener un impacto en el diagnóstico de estos pacientes.

Aún las pruebas de coagulación más rutinarias son complejas por naturaleza ${ }^{22}$. Por esta razón deben idealmente realizarse en laboratorios de referencia, con personal capacitado adecuadamente, con equipos y reactivos verificados, y métodos comparables y confiables. Esta revisión resalta la importancia de estandarizar protocolos de recolección, tratamiento y manejo de muestras a nivel nacional e internacional, con el fin de reducir la variabilidad que aporta al estudio de un paciente con HAA la fase pre-analítica de las pruebas diagnósticas.

\section{Referencias}

1. Mulliez SMN, Vantilborgh A, Devreese KMJ. Acquired hemophilia: A case report and review of the literature. Int J Lab Hematol 2014; 36 (3): 398-407.

2. Franchini M, Mannucci PM. Acquired haemophilia A: a 2013 update. Thromb Haemost 2013; 110 (6): 1114-20.

3. Coppola A, Favaloro EJ, Tufano A, Di Minno MND, Cerbone AM, Franchini M. Acquired Inhibitors of coagulation factors: Part I-acquired hemophilia a. Semin Thromb Hemost 2012; 38 (5): 433-46.

4. Franchini M, Mannucci PM. Inhibitors of propagation of coagulation (factors VIII, IX and XI): A review of current therapeutic practice. Br J Clin Pharmacol 2011; 72 (4): 553-62.

5. Cohen AJ, Kessler CM. Acquired inhibitors. Baillieres Clin Haematol 1996; 9 (2): 331-54.

6. Franchini M, Castaman G, Coppola A, Santoro C, Zanon E, Di Minno G, et al. Acquired inhibitors of clotting factors: AICE recommendations for diagnosis and management. Blood Transfus 2015; 13 (3): 498-513.

7. Knoebl P, Marco P, Baudo F, Collins P, Huth-Kühne A, Nemes L, et al. Demographic and clinical data in acquired hemophilia A: Results from the European Acquired Haemophilia Registry (EACH2). J Thromb Haemost 2012; 10 (4): 622-31.

8. Shander A, Walsh C, Bailey H, Cromwell C. Acquired hemophilia presenting as profound hematuria: Evaluation, diagnosis, and management of elusive cause of bleeding in the emergency department setting. J Emerg Med 2013; 45 (1): e1-6.

9. Ceresetto JM, Duboscq C, Fondevila C, Pinto MT. Hemofilia adquirida (inhibidor adquirido del factor VIII). Med 2015; 75 (4): 231-8.

10. Bonfanti C, Crestani S, Frattini F, Sissa C, Franchini M. Role of rituximab in the treatment of postpartum acquired haemophilia A: a systematic review of the literature. Blood Transfus 2015; 13: 396-400.

11. Mingot-Castellano ME, Núñez R, Rodríguez-Martorell FJ. Hemofilia adquirida: epidemiología, clínica, diagnóstico y tratamiento. Med Clin (Barc) 2017; 148 (7): 314-22.

12. Favaloro EJ, Lippi G. Preanalytical issues that may cause misdiagnosis in haemophilia and von Willebrand disease. Haemophilia 2018; 24 (2): 198-210.

13. Hoffman M, Dargaud Y. Mechanisms and monitoring of bypassing agent therapy. J Thromb Haemost 2012; 10 (8): 1478-85.

14. Hoffman M, Monroe DM. A cell-based model of hemostasis. Thromb Haemost 2001; 85 (6): 958-65.

15. Zdziarska J, Musiał J. Acquired hemophilia A: An un- 
derdiagnosed, severe bleeding disorder. Pol Arch Med Wewn 2014; 124 (4): 200-6.

16. Dimichele DM. Inhibidores en Hemofilia: Cuarta edición. 2008; (7).

17. Kasper CK. Diagnóstico y tratamiento de inhibidores de los factores VIII y XI. Fed Mund Hemofilia 2004; (34).

18. Shetty S, Bhave M, Ghosh K. Acquired hemophilia A: Diagnosis, aetiology, clinical spectrum and treatment options. Autoimmun Rev 2011; 10 (6): 311-6.

19. Kruse-Jarres R, Kempton CL, Baudo F, Collins PW, Knoebl P, Leissinger CA, et al. Acquired hemophilia A: Updated review of evidence and treatment guidance. Am J Hematol 2017; 92 (7): 695-705.

20. Duboscq C, Ceresetto JM, Arias M, Forastiero R. Detección de inhibidor adquirido específico de factor VIII Diagnosis of acquired inhibitor against factor VIII. Acta Bioquim clin Latinoam 2016; 50 (2).

21. Kershaw G, Orellana D. Mixing tests: Diagnostic aides in the investigation of prolonged prothrombin times and activated partial thromboplastin times. Semin Thromb Hemost 2013; 39 (3): 283-90.

22. Giangrande P. Treatment of Hemophilia: Acquired Hemophilia. Revised edition. World Fed Hemoph 2012; (38): 1-7.
23. Batty P, Moore GW, Platton S, Maloney JC, Palmer B, Bowles L, et al. Diagnostic accuracy study of a factor VIII ELISA for detection of factor VIII antibodies in congenital and acquired haemophilia A. Thromb Haemost 2015; 114 (4): 804-11.

24. Tiede A, Werwitzke S, Scharf RE. Laboratory Diagnosis of Acquired Hemophilia A: Limitations, Consequences, and Challenges. Semin Thromb Hemost 2014; 40 (7): 803-11.

25. Dardikh M, Meijer P, Van Der Meer F, Favaloro EJ, Verbruggen B. Acquired functional coagulation inhibitors: Review on epidemiology, results of a wet-workshop on laboratory detection, and implications for quality of inhibitor diagnosis. Semin Thromb Hemost 2012; 38 (6): 613-21.

26. Favaloro EJ, Lippi G, Adcock DM. Preanalytical and postanalytical variables: The leading causes of diagnostic error in hemostasis? Semin Thromb Hemost 2008; 34 (7): 612-34.

27. Baluwala I, Favaloro EJ, Pasalic L. Therapeutic monitoring of unfractionated heparin-trials and tribulations. Expert Rev Hematol 2017; 10 (7): 595-605.

28. Talapatra A, Nash MJ, Hay CRM, Thachil J. Acquired haemophilia: An easy diagnosis to miss in a patient taking warfarin. Acute Med 2015; 14 (3): 122-4. 